\title{
Multi-locus variable-number tandem repeat analysis of Chinese Brucella strains isolated from 1953 to 2013
}

Guo-Zhong Tian ${ }^{1 \dagger}$, Bu-Yun Cui ${ }^{1 \dagger}$, Dong-Ri Piao ${ }^{1}$, Hong-Yan Zhao ${ }^{1}$, Lan-Yu Li ${ }^{1}$, Xi Liu' ${ }^{1}$, Pei Xiao ${ }^{2}$, Zhong-Zhi Zhao ${ }^{3}$, Li-Qing $\mathrm{Xu}^{3}$, Hai Jiang ${ }^{1^{*}}$ and Zhen-Jun $\mathrm{Li}^{1^{*}}$

\begin{abstract}
Background: Brucellosis was a common human and livestock disease caused by Brucella strains, the category B priority pathogens by the US Center for Disease Control (CDC). Identified as a priority disease in human and livestock populations, the increasing incidence in recent years in China needs urgent control measures for this disease but the molecular background important for monitoring the epidemiology of Brucella strains at the national level is still lacking.

Methods: A total of 600 Brucella isolates collected during 60 years (from 1953 to 2013) in China were genotyped by multiple locus variable-number tandem repeat analysis (MLVA) and the variation degree of MLVA11 loci was calculated by the Hunter Gaston Diversity Index (HGDI) values. The charts and map were processed by Excel 2013, and cluster analysis and epidemiological distribution was performed using BioNumerics (version 5.1).

Results: The 600 representative Brucella isolates fell into 104 genotypes with 58 singleton genotypes by the MLVA11 assay, including B. melitensis biovars 2 and 3 (five main genotypes), B. abortus biovars 1 and 3 (two main genotypes), $B$. suis biovars 1 and 3 (three main genotypes), and $B$. canis (two main genotypes) respectively. While most B. suis biovar 1 and biovar 3 were respectively found in northern provinces and southern provinces, B. melitensis and B. abortus strains were dominant in China. Canine Brucellosis was only found in animals without any human cases reported. Eight Brucellosis epidemic peaks emerged during the 60 years between 1953 and 2013: 1955 - 1959, 1962 - 1969, 1971 - 1975, 1977 - 1983, 1985 - 1989, 1992 - 1997, 2000 - 2008 and 2010 - 2013 in China.
\end{abstract}

Conclusions: Brucellosis has its unique molecular epidemiological patterns with specific spatial and temporal distribution according to MLVA.

Trial registration: IDOP-D-16-00101.

Keywords: Brucella, MLVA, Molecular epidemiology

\section{Multilingual abstracts}

Please see Additional file 1 for translation of the abstract into the five official working languages of the United Nations.

\footnotetext{
* Correspondence: jianghai@icdc.cn; lizhenjun@icdc.cn

${ }^{\dagger}$ Equal contributors

${ }^{1}$ State Key Laboratory for Infectious Disease Prevention and Control, Collaborative Innovation Center for Diagnosis and Treatment of Infectious Diseases, National Institute for Communicable Disease Control and Prevention, Chinese Center for Disease Prevention and Control, Beijing 102206, China

Full list of author information is available at the end of the article
}

\section{Background}

Brucellosis is one of the world's most widespread zoonotic diseases. It is also seen as a reemerging infectious disease because of increasing incidence in recent years. It also is identified as a priority disease needing urgent control measures in human and livestock populations in China [1-7]. A total of 43486 confirmed human cases were reported in 2013 (annual incidence of 3.3 cases/100 000 population) in China, and 57222 cases were reported in 2014. Brucellosis has been found in all China.

Previous studies have confirmed that the multiple locus variable-number tandem repeat analysis (MLVA) is 
a useful tool for identifying and genotyping of strains and for epidemiological trace-back investigations [8]. It was also applied in Brucella [5-7]. The objectives of this study was to analyze the genotyping of 600 Brucella isolates, which were isolated from 1953 to 2013 in China by multiple locus variable-number tandem repeat analysis (MLVA) in order to more comprehensively understand the patterns of molecular epidemiology of Brucellosis in China, to trace the flow direction and variation of Brucella, and to provide reference information for the prevention and control of Brucellosis in China.

\section{Methods}

\section{Brucella isolates data}

There were 2620 human and animal field Brucella isolates, which were collected from 29 provinces in China from 1953 to 2013. These strains came from the provincial CDC which were isolated and cultured from blood and tissues (such as liver and spleen). These isolates were identified as Brucella based on distinct host specificity, pathogenicity, and minor phenotypic differences based on serotyping, phage typing, fuchsin and thionin dye sensitivity, $\mathrm{CO}_{2}$ requirement, $\mathrm{H}_{2} \mathrm{~S}$ production and metabolic properties [9]. They were dried and preserved in the $-80{ }^{\circ} \mathrm{C}$ refrigerator in our laboratory. The stratified sampling method was used to select the number of Brucella isolates for this study. The definition of strata was Brucella bioytping. The selected proportion was $20.0 \%$ in each strata. $22.9 \%(600 / 2$ 620) of Brucella isolates were selected for this study.

\section{Molecular typing methods}

Total genomic DNA was individually extracted from the 600 representative strains and MLVA was performed as described previously [10, 11]. The MLVA11 assay involved 11 loci including Bruce06, Bruce08, Bruce11, Bruce12, Bruce18, Bruce19, Bruce21, Bruce42, Bruce43, Bruce45, and Bruce55. A total of 11 PCR primers were labeled with $5^{\prime}$-fluorescent 6-FAM and PCR products were resolved by capillary electrophoresis on an ABI Prism 3130 automated fluorescent capillary DNA sequencer (Applied Biosystems). The PCR fragment sizes were converted to repeat units by following the published allele numbering system (http://mlva.u-psud.fr, Brucella support website for MLVA typing). All data were analyzed by using BioNumerics (version 5.1, Applied Maths, Belgium). Cluster analysis was based on the categorical coefficient and unweighted pair group method using arithmetic averages (UPGMA) method. The variation degree of MLVA11 loci was calculated by Hunter Gaston Diversity Index (HGDI) values. The charts and map were produced by Excel 2013.

\section{Results}

Genetic diversity of MLVA-11 loci and cluster analysis

The Hunter Gaston Diversity Index (HGDI) value for each MLVA-11 locus was calculated for the 600 Brucella isolates (see Table 1). Of the 600 Brucella isolates identified by the MLVA11 assay. B. melitensis was the majority with 398 strains (66.3\%), followed by $97(16.2 \%)$ of $B$. abortus, 58 (9.7\%) of B. suis, and $47(7.8 \%)$ of $B$. canis. Biovars 2 and 3 were dominant (99.0\%, 394 strains) in B. melitensis with only four strains (1.0\%) of biovar 1. Biovar 3 (75 strains) was the majority (77.3\%) in B. abortus, with $17.5 \%$ of biovar 1 and five $5.2 \%$ of biovar 9 . Biovar 1 and biovar 3 were almost half and half (48.3\%) in B. suis with 28 strains each, and the remaining two strains (3.4\%) were of biovar 2 (Additional file 2: Table S1).

The MLVA11 assay profiled the 600 Brucella isolates into 104 genotypes with 58 singleton genotypes (Fig. 1). The number of strains for each genotype was shown in Fig. 2. The largest group (59 genotypes) belonged to the 398 $B$. melitensis isolates, with 23,17 and 5 genotypes representing $B$. abortus, $B$. suis and B. canis strains respectively (Fig. 1). These strains formed two branches (1 and 2) with 12 subclusters in total. Branch 1 was composed of B. abortus and B. melitensis whereas B. suis (biovars 1,2 and 3) and $B$. canis belonged to branch 2 . Cluster A, the largest with 394 B. melitensis biovars 2 and 3 isolates, were further divided into 4 subclusters (A1, A2, A3 and C) with a percentage of genetic similarity coefficient of $56.8 \%$. Cluster $\mathrm{B}$, including 97 B. abortus isolates, were further divided into four subclusters (B1, B2, B3 and B4) with a percentage of genetic similarity coefficient of $75.0 \%$. Cluster $\mathrm{C}$ was small,

Table $1 \mathrm{HGDI}$ values of MLVA-11 loci for Brucella strains

\begin{tabular}{lllll}
\hline Locus & Diversity Index $(n=600)$ & Confidence Interval & $\mathrm{K}$ & Max (pi) \\
\hline Bruce19 & 0.867 & $0.843-0.892$ & 16 & 0.200 \\
Bruce12 & 0.758 & $0.713-0.802$ & 10 & 0.333 \\
Bruce18 & 0.703 & $0.646-0.760$ & 7 & 0.457 \\
Bruce06 & 0.62 & $0.548-0.692$ & 6 & 0.552 \\
Bruce11 & 0.567 & $0.465-0.669$ & 8 & 0.638 \\
Bruce08 & 0.533 & $0.441-0.624$ & 5 & 0.648 \\
Bruce21 & 0.513 & $0.424-0.603$ & 6 & 0.657 \\
Bruce43 & 0.512 & $0.424-0.600$ & 4 & 0.657 \\
Bruce42 & 0.503 & $0.406-0.601$ & 6 & 0.676 \\
Bruce55 & 0.395 & $0.290-0.501$ & 4 & 0.762 \\
Bruce45 & 0.364 & $0.271-0.458$ & 4 & 0.771
\end{tabular}

Hunter Gaston Diversity Index (HGDI) was used to measure the variation of multiple locus variable-number tandem repeat locus, which ranges from 0.0 (no diversity) to 1.0 (complete diversity); Confidence Interval is Precision of the Diversity Index and expressed as 95\% upper and lower boundaries; K: Number of different repeats present at this locus in this sample set; Max (pi): Fraction of samples that have the most frequent repeat number in this locus (ranging from 0.0 to 1.0$) ; n$ : The number of the isolates according to the considered locus 


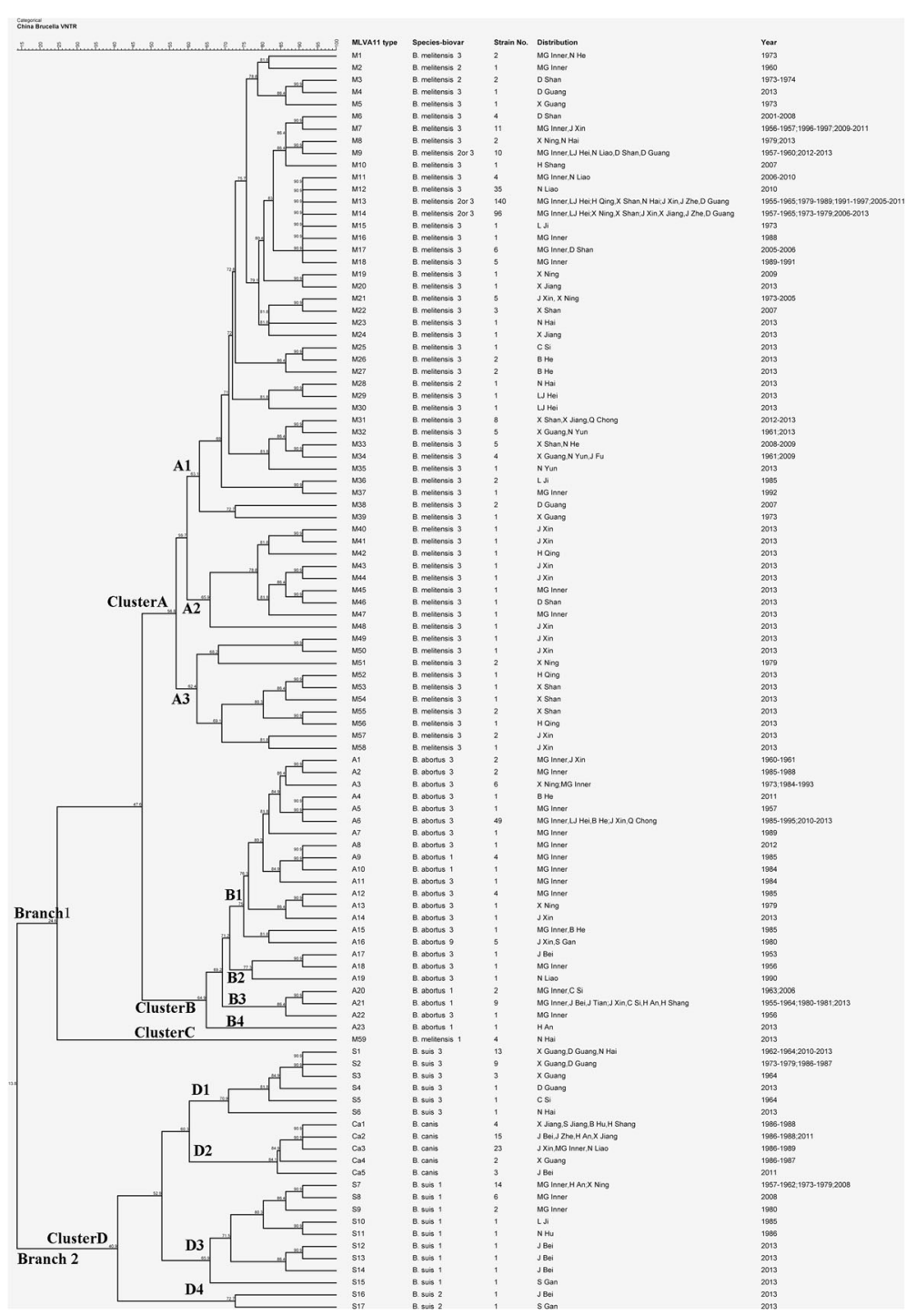

Fig. 1 Cluster analysis by MLVA11 genotyping of the 600 Brucella strains from 1953 - 2013 in China. The cluster analysis was performed using the category coefficient and UPGMA (BioNumerics 5.1). B He:Hebei, B Hu:Hu Bei,C Si:Sichuan,D Guang:Guangdong,D Shan:Shandong, H An:Anhui,H Qing:Qinghai, H Shang:Shanghai, J Bei:Beijing, J Fu:Fujian, J Xin:Xinjiang,J Zhe:Zhejiang,L Ji:Jilin,L H Hei:Heilongjiang, MG Inner:Inner Mongolia, N Hai:Hainan,N He:Henan, N He:Henan, N Hu:Hunan,N Liao:Liaoning, N Yun:Yunnan,S Gan:Gansu, Tianjin:J Tian, X Guang:Guangxi,X Jiang:Jiangxi, X Ning:Ningxia,X Shan:Shanxi

containing only four strains of $B$. melitensis biovar 1 . Branch 2 contained four clusters (D1, D2, D3 and D4) with a genetic similarity coefficient of $40.9 \%$. The 58 B. suis isolates were scattered among clusters D1, D3 and D4. The 47 B. canis isolates together with the 28 B. suis biovar 3 isolates formed cluster D2 with a percentage of genetic similarity coefficient of $56.8 \%$ (Fig. 1).

Among the 104 genotypes were 12 main genotypes with more than two thirds $70.7 \%(424 / 600)$ of the 600 Brucella isolates. Specifically, 5 genotypes (M7, M9, M12, M13 and M14) accounted for 73.4\% (292/398) of the B. melitensis strains. Two genotypes (A6 and A21) encompassed 59.8\% (58/97) of B. abortus. Genotypes S1,
S2, and S7 contained $62.1 \%(36 / 58)$ of the B. suis isolates whereas genotypes $\mathrm{Ca} 2$ and $\mathrm{Ca} 3$ covered $80.9 \%$ (38/47) of the B. canis strains (Fig. 2).

The 600 strains could also be stratified by their geographical origin and time frame of isolation as shown in Fig. 3. All strains were from 4 regions: northern, northwestern, central and southern with details as follows. From the northern region, Inner Mongolia contributed the most nationwide $(25.2 \%, 151 / 600)$, followed by Liaoning $(6.7 \%, 40 / 600)$ and Heilongjiang $(2.7 \%, 16 /$ $600)$ provinces. The numbers for the northwestern region were Xinjiang $(7.3 \%, 44 / 600)$, Ningxia $(11.8 \%, 71 /$ $600)$ and Shanxi $(5.2 \%, 31 / 600)$. The central region were 


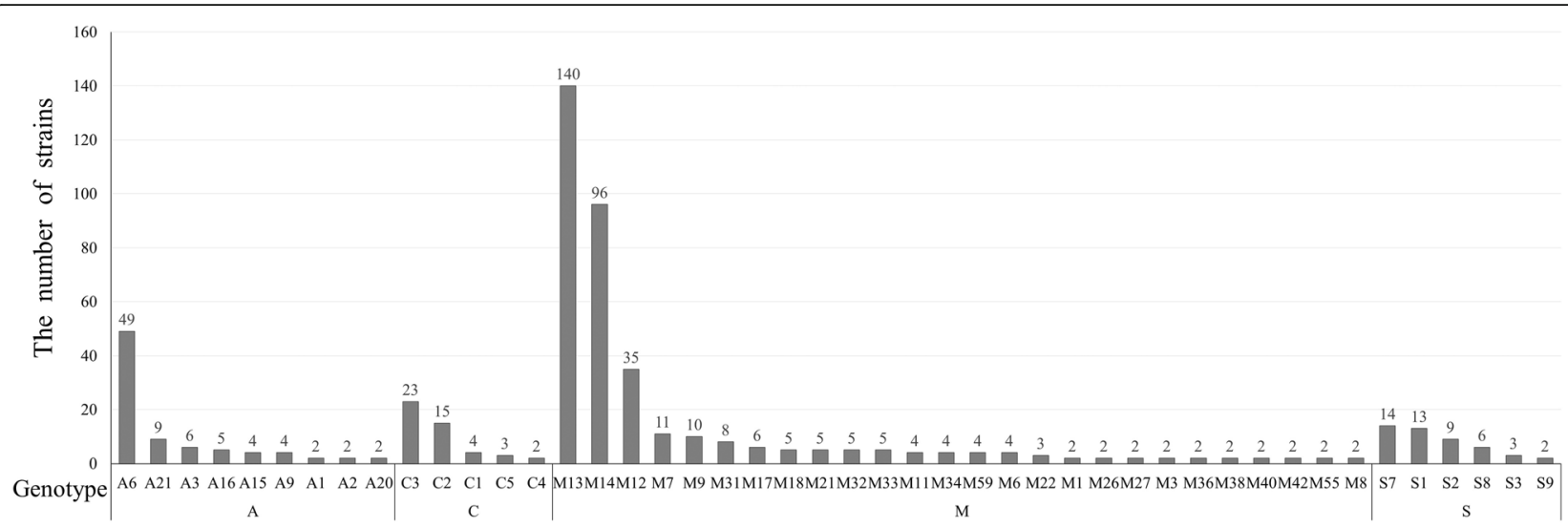

Fig. 2 The relationship between the numbers of isolated strains and MLVA genotypes. The 58 singleton genotypes were not shown on the chart

represented by Hebei $(2.7 \%, 16 / 600)$, Beijing (6.2\%, 37/ $600)$, Shandong $(2.5 \%, 15 / 600)$, Jiangxi $(2.8 \%, 17 / 600)$ and Zhejiang $(2.0 \%, 12 / 600)$ provinces. Representing the southern region were Guangdong $(8.2 \%, 49 / 600)$, Guangxi $(3.8 \%, 23 / 600)$ and Hainan $(2.5 \%, 15 / 600)$ provinces.

The time frame of strain isolation with more than 15 strains in a year was: 1973 (3.7\%, 22/600), 1979 (4.0\%, 24/600), 1985 (2.7\%, 16/600), 1988 (3.2\%, 19/600), 2006 (2.8\%, 17/600), 2007 (3.0\%, 18/600), 2008 (3.3\%, 20/600), 2009 (4.7\%, 28/600), 2010 (10.2\%, 61/600), 2011 (8.0\%, 48/600), 2012 (3.7\%, 22/600) and 2013 (15.8\%, 94/600), and unkown year $(3.2 \%, 19 / 600)$ (Fig. 4).

The 600 Brucella strains were respectively isolated from blue sheep and pig (one strain each), camel (two), deer (three), yak (four), goat (12), swine (19), dog (47), sheep (81), cattle (90), human (272) and unknown (68).
The time frame and geographic regions of the isolated strains

A total of 398 B. melitensis isolates were divided into four clusters (A1, A2, A3, C) with a percentage of genetic similarity coefficient of $62.4 \%$. Cluster A1 (372 strains belonging to $B$. melitensis biovars 2 and 3 ) were found in Inner Mongolia, Heilongjiang, Liaoning, Shandong, Guangdong, Xinjiang, Ningxia, Qinghai, Shanxi, Henan, Jiangxi, Guangxi, Fujian and Hainan provinces across years of 1955 - 1965, 1973 - 1979, 1991 - 1997, 2005 - 2008, and 2010 - 2013. Cluster A2 including 9 strains were isolated from Xinjiang, Inner Mongolia, Shandong and Qinghai provinces in 2013. Cluster A3 (13 isolates) were isolated from Xinjiang, Shanxi, Ningxia and Qinghai provinces in 2013 whereas cluster C (four isolates) was from Hainan province in 2013 (Fig. 5).

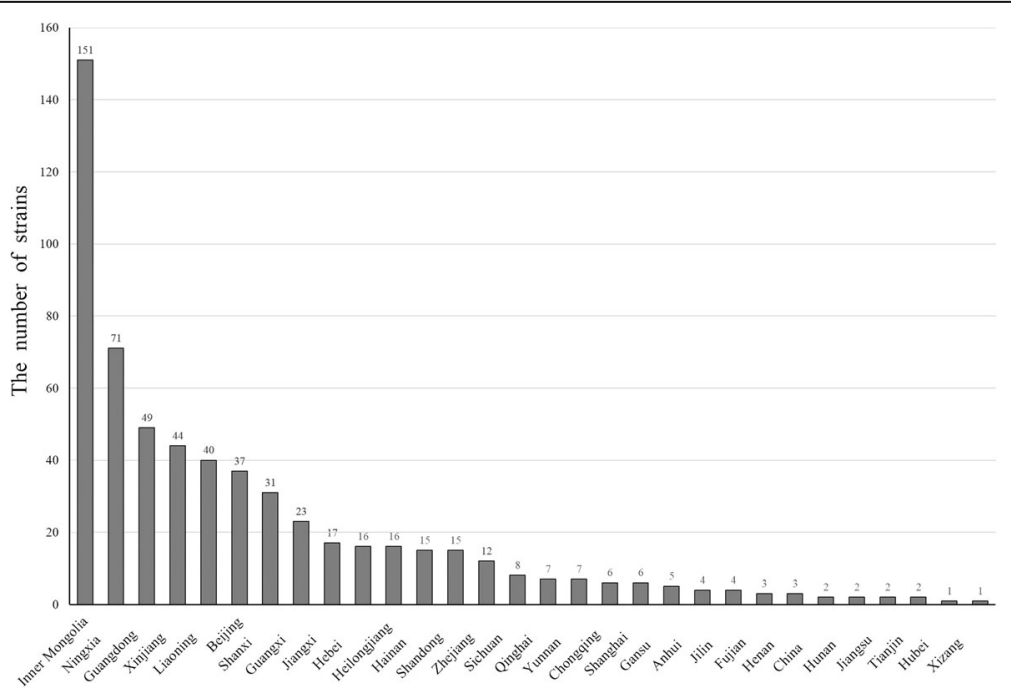

Fig. 3 The relationship between numbers of isolated strains and isolated origins. The numbers marked in solid lines (isolated years) and dotted lines (isolated origins) represent the number of isolates that were isolated by their origins and year of isolation 


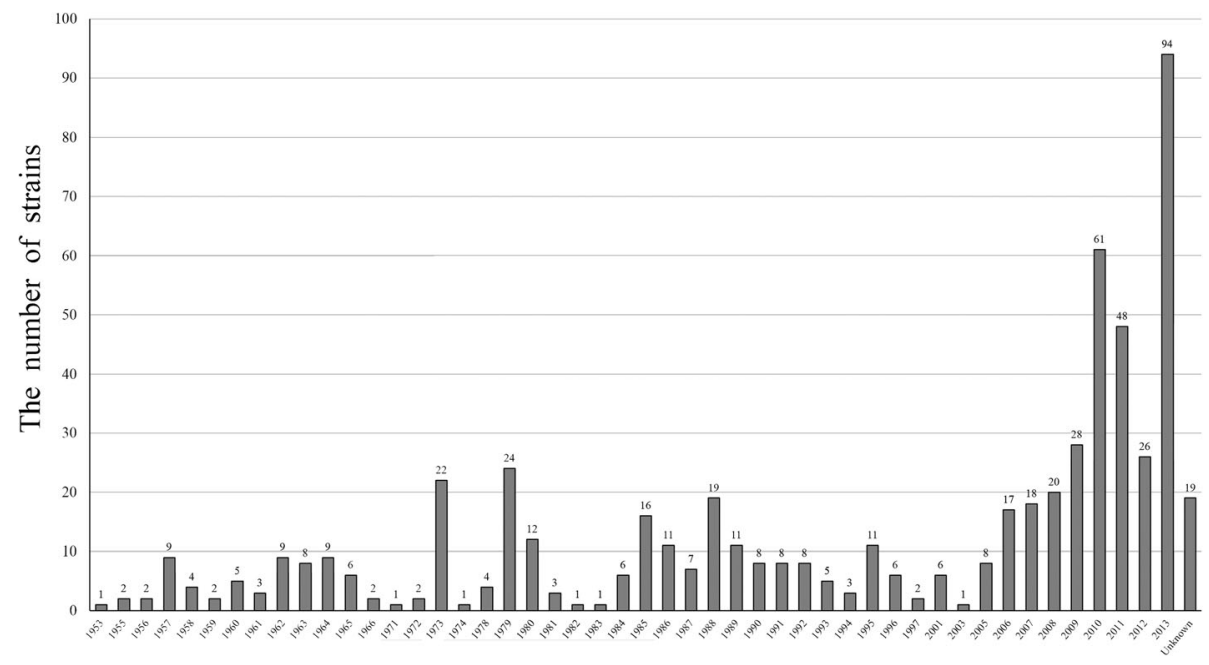

Fig. 4 The relationship between the numbers of isolated strains and the isolated years

The four clusters (B1, B2, B3 and B4) consisted of 97 $B$. abortus isolates had a percentage of genetic similarity coefficient of $75.0 \%$. The dominating cluster B1 included $81 \mathrm{~B}$. abortus biovars 1 and 3 isolates from Inner Mongolia, Heilongjiang, Hebei, Xinjiang and Chongqing provinces during 1973 - 1979, 1984 - 1985 and 2010 2013. The three $B$. abortus biovar 3 isolates comprising cluster B2 were from Liaoning, Inner Mongolia and Beijing provinces during 1953 - 1956. By contrast of cluster B4 with just a single isolate from Anhui province in 2013, cluster B3 had 12 strains of B. abortus biovar 1 and 3 isolates from Xinjiang, Inner Mongolia, Tianjin, Sichuan, Anhui and Shanghai provinces during 1955 1964, 1980 - 1981 and 2013 (Fig. 5).

The D1, D3, D4 clusters (58 B. suis isolates) had a percentage of genetic similarity coefficient of $65.9 \%$. Cluster D1 (28 biovar 3 B. suis strains) were isolated from Guangdong, Guangxi and Hainan provinces during 1962 - 1964, 1973 - 1979, 1986 - 1987 and $2010-$ 2013. The second included 28 B. suis biovar 1 isolates,

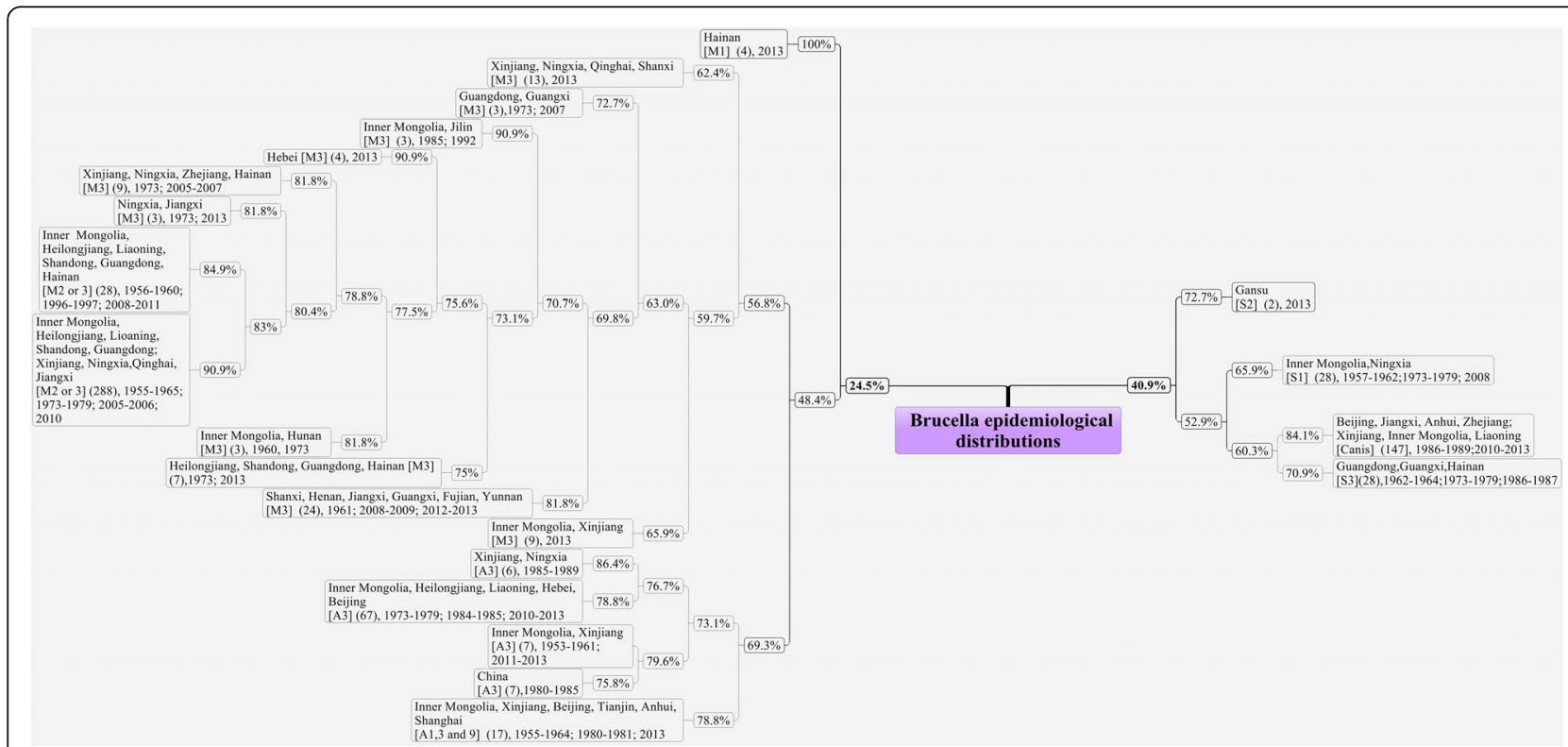

Fig. 5 The epidemiological distributions of the 600 Brucella strains by MLVA11 genotyping. Note: "24.5\%", the percentage of genetic similarity coefficient was 24.5\%; "Hainan [M1] (4), 2013": "[M1]", Brucella melitensis biotype 1; "(4)", there was 4 strains with Brucella melitensis biotype 1; "2013", the isolation year was 2013. "[M2 or 3]", Brucella melitensis biotype 2 and 3; "[A1]", Brucella Abortus biotype 1; "[A3]", Brucella Abortus biotype 3; "[A9]", Brucella Abortus biotype 9; "[S1]", Brucella suis biotype 1; "[S2]", Brucella suis biotype 2; "[S3]", Brucella suis biotype 3; "[Canis]", Brucella canis 
representing cluster D3, were from Inner Mongolia, Ningxia, Beijing, Jilin and Hunan provinces collected during 1957 - 1962, 1973 - 1979, 1986 - 1987, 2008 and 2013. D4 was a small cluster with only two biovar 2 isolates of B. suis from Gansu province in 2013 (Fig. 5).

With a percentage of genetic similarity coefficient of $84.1 \%$, cluster D2 (47 B. canis isolates) was consisted of genotypes $\mathrm{Ca} 2$ and $\mathrm{Ca} 3$. Genotype $\mathrm{Ca} 2(31.9 \%, 15 / 47)$ were isolated from Zhejiang, Anhui and Jiangxi provinces during 1986 - 1988 and 2010 - 2011. Genotype Ca3 (48.9\%, 23/ 47) were collected from Xinjiang, Inner Mongolia and Liaoning provinces during 1986 - 1989 (Fig. 5).

\section{Discussion}

For many years, phenotypic characterization for Brucella strains was the only feasible way to provide epidemiological data with the disadvantage of lacking information related to their molecular background $[8,12]$. Now with molecular tools, more options are available.

Although having higher discriminatory power than the MLVA11 assay, the MLVA16 assay with a set of 16 repeat loci is better for teasing out the difference of closely related isolates at fine-scale resolution whereas the MLVA11 assay is more suitable to analyze the phylogenetic and epidemiological relationships of Brucella strains because of its lesser degree of variation degree (HGDI values) [11, 13-18]. In accord with this notion, the genotyping results of MLVA11 assay have been consistent with those of MLVA71 assay genotyping [19], Brucella-specific IS711 element analysis [13], and wholegenome-based phylogeny studies $[15,16]$. Therefore, we chose the MLVA11 assay to study the molecular epidemiology of Brucella isolates from China.

The results of our cluster analysis using the MLVA11 assay were meaningful in terms of the isolation time frame, host and region of these strains. For the past 60 years the dominant Brucella species and biovars were B. abortus biovars 1 and 3, B. melitensis biovars 2 and 3, B. suis biovar 1 and 3 , and B. canis. The cluster analysis results of the 600 Brucella isolates correlated well with their genetic variation patterns and the Brucellosis epidemics. For instances, before 1980 most B. abortus biovars 1 and 3 isolates were seen in the northern region such as Inner Mongolia, Xinjiang, Gansu and Ningxia provinces. However, after 2000 B. abortus strains have emerged in the central and southern regions like Hebei, Beijing, Chongqing and Zhejiang provinces. According to results of our MLVA analysis, there were eight epidemic periods across years of 1955 - 1959, 1962 - 1969, 1971 - 1975, 1977 - 1983, 1985 - 1989, 1992 - 1997, $2000-2008$ and $2010-2013$ (Figs. 1, 5 and 6) with each peak at years of 1958, 1966, 1973, 1980, 1987, 1996, 2007 and 2013. Most of the involved livestock were either raised or processed in the northern and northwestern regions in China. Results of the MLVA analysis (Figs. 1, 5 and 6) suggested that there were three predominant but

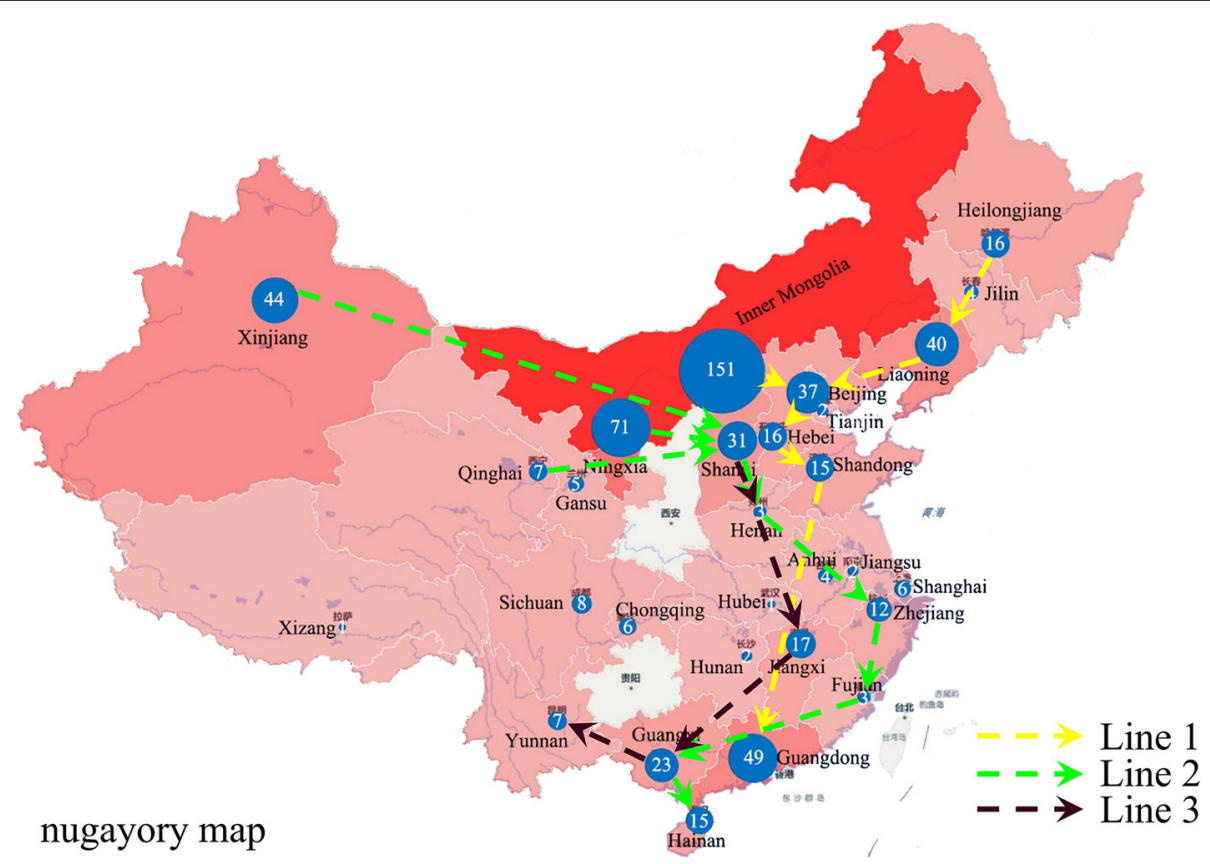

Fig. 6 The sketch map of the 600 Brucella strains from different provinces in this study. Line 1 (yellow line): Inner Mongolia, Heilongjiang, Liaoning-Shandong- Hebei- Beijing-Guangdong provinces. Line 2 (green line): Xinjiang, Ningxia, Qinghai-Shanxi-Henan-Zhejiang-Guangxi-FujianHainan provinces. Line 3 (black line) Shanxi-Henan-Jiangxi-Guangxi-Yunnan provinces. The size of the blue circle represented the total number of the isolated strains (in a circle). The sketch map does not represent an administrative map in the legal sense 
different lines of Brucella transmission in China. The first line (Line 1) involved Inner Mongolia, Heilongjiang, Liaoning, Shandong, Hebei, Beijing and Guangdong provinces. The second line included places in Xinjiang, Ningxia, Qinghai, Shanxi, Henan, Zhejiang, Guangxi, Fujian and Hainan provinces (Line 2). And the third line was represented by Shanxi, Henan, Jiangxi, Guangxi and Yunnan provinces (Line 3) (Fig. 6). As to the species and biovar, most $B$. suis biovar 1 isolates were from the northern region including Inner Mongolia and Ningxia provinces while the majority of the $B$. suis biovar 3 isolates were from the southern region, such as Guangdong and Guangxi provinces.

Considering the finding that most of the cluster A1 strains $(93.5 \%$ of the B. melitensis biovars 2 and 3, with a percentage of genetic similarity coefficient of $62.4 \%$ ) have been existing in different provinces for a long time (from 1953 to 2013) whereas clusters A2 and A3 isolates started to appear in 2013 in Inner Mongolia, Xinjiang and Shanxi provinces (Fig. 1), questions arise such as whether these cluster A2 and A3 strains are variants of other biovars, or have they been causing diseases all these years but without being isolated, what is its impact on trend of Brucellosis in China, and so on. All such questions remain to be answered in the future.

\section{Conclusions}

Taken together, our study showed the MLVA is a reliable monitoring method for the molecular epidemiology of Brucellosis relating to strain phylogeny and the regional and time distribution of the disease, potentially valuable for predicting the epidemic trend of Brucellosis in China.

\section{Additional files}

Additional file 1: Multilingual abstract in the five official working languages of the United Nations. (PDF $972 \mathrm{~kb}$ )

Additional file 2: Table S1. MLVA-11 genotyping and the data of 600 Brucella strains in China. (XLS $166 \mathrm{~kb}$ )

\footnotetext{
Acknowledgements

This study was supported by grants from the Chinese National Programs for High Technology Research and Development: Lab Infectious Materials and Biological Risk Source Key Technology and Product Research (No. 2014AA021404) and the National Nature Science Foundation (No. 81271900). We would like to thank the staff who were involved and contributed to the data and Brucella isolate collection across the country. We are also grateful to all the staff of the Department of Brucellosis, National Institute for Communicable Disease Control and Prevention, Chinese Center Disease Control and Prevention for their contribution to this research. We are grateful to Professor Xin-He Lai, Department of Pediatrics \& Insitute of Translational Medicine, Wenzhou Medical University, for proofreading the final version of the manuscript.
}

Availability of data and materials

Please contact the corresponding author for data requests.

\section{Authors' contributions}

$\mathrm{GT}, \mathrm{BC}$ and $\mathrm{ZL}$ proposed the idea and designed the study. GT, DP, HZ, LL, XL, PX, ZZ and LX characterized the Brucella strains by classical biotyping methods. GT, BC, HJ, PX conducted the MLVA analysis. GT, BC, HJ and ZL performed the statistical analysis. All authors read and approved the final version of the submitted manuscript.

\section{Competing interests}

The authors declared that they have no competing interests.

Consent for publication

Not applicable.

Ethics approval and consent to participate

Ethical approval was received from National Institute for Communicable Disease Control and Prevention, Chinese Center Disease Control and

Prevention. Ethical Committee Approval Notice ICDC-2015007.

\section{Author details}

'State Key Laboratory for Infectious Disease Prevention and Control, Collaborative Innovation Center for Diagnosis and Treatment of Infectious Diseases, National Institute for Communicable Disease Control and Prevention, Chinese Center for Disease Prevention and Control, Beijing 102206, China. ${ }^{2}$ National Institute of Occupational Health and Poison Control, Chinese Center for Disease Prevention and Control, Beijing 100050, China. ${ }^{3}$ Department of Brucellosis Prevention and Control, Qinghai Institute for Endemic Disease Prevention and Control, Xining 811602, China.

Received: 9 May 2016 Accepted: 29 March 2017

Published online: 02 May 2017

\section{References}

1. Pappas G, Papadimitriou P, Akritidis N, Christou L, Tsianos EV. The new global map of human Brucellosis. Lancet Infect Dis. 2006;6:91-9.

2. Seleem MN, Boyle SM, Sriranganathan N. Brucellosis: a re-emerging zoonosis. Vet Microbiol. 2010;140:392-8.

3. Deqiu S, Donglou X, Jiming Y. Epidemiology and control of Brucellosis in China. Vet Microbiol. 2002;90:165-82.

4. Zhang WY, Guo WD, Sun SH, Jiang JF, Sun HL, Li SL, et al. Human brucellosis, inner Mongolia. China Emerg Infect Dis. 2010;16:2001-3.

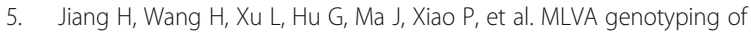
Brucella melitensis and Brucella abortus isolates from different animal species and human and identification of Brucella suis vaccine strain S2 from cattle in China. PLoS One. 2013;8:e76332.

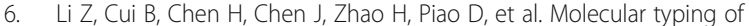
Brucella suis collected from 1960s to 2010s in China by MLVA and PFGE. Biomed Environ Sci. 2013:26:504-8.

7. Di D, Cui B, Wang H, Zhao H, Piao D, Tian L, et al. Genetic polymorphism characteristics of Brucella canis isolated in China. PLoS One. 2014:9:e84862.

8. Al Dahouk S, Flèche PL, Nöckler K, Jacques I, Grayon M, Scholz HC, et al. Evaluation of Brucella MLVA typing for human Brucellosis. J Microbiol Methods. 2007:69:137-45.

9. Alton G, Jones L. Laboratory techniques in Brucellosis. Monogr Ser World Health Organ. 1967;55:1-92.

10. Marianelli C, Graziani C, Santangelo C, Xibilia MT, Imbriani A, Amato R, et al. Molecular epidemiological and antibiotic susceptibility characterization of Brucella isolates from human in Sicily. Italy J Clin Microbiol. 2007;45:2923-8.

11. Maquart M, Le Flèche P, Foster G, Tryland M, Ramisse F, Djønne B, et al. MLVA-16 typing of 295 marine mammal Brucella isolates from different animal and geographic origins identifies 7 major groups within Brucella ceti and Brucella pinnipedialis. BMC Microbiol. 2009;9:145.

12. Whatmore AM. Current understanding of the genetic diversity of Brucella, an expanding genus of zoonotic pathogens. Infect Genet Evol. 2009;9:1168-84.

13. Audic S, Lescot M, Claverie JM, Cloeckaert A, Zygmunt MS. The genome sequence of Brucella pinnipedialis B2/94 sheds light on the evolutionary history of the genus Brucella. BMC Evol Biol. 2011;11:200.

14. Chen $Y$, Zhen Q, Wang Y, Xu J, Sun Y, Li T, et al. Development of an extended multilocus sequence typing for genotyping of Brucella isolates. J Microbiol Methods. 2011;86:252-4. 
15. Foster JT, Beckstrom-Sternberg SM, Pearson T, Beckstrom-Sternberg JS, Chain PS, Roberto FF, et al. Whole-genome-based phylogeny and divergence of the genus Brucella. J Bacteriol. 2009;191:2864-70.

16. Foster JT, Price LB, Beckstrom-Sternberg SM, Pearson T, Brown WD, Kiesling DM, et al. Genotyping of Brucella species using clade specific SNPs. BMC Microbiol. 2012;12:110.

17. Garofolo G, Di Giannatale E, De Massis F, Zilli K, Ancora M, Cammà C, et al. Investigating genetic diversity of Brucella abortus and Brucella melitensis in Italy with MLVA-16. Infect Genet Evol. 2013;19:59-70.

18. Dorneles EM, Santana JA, Alves TM, Pauletti RB, Mol JP, Heinemann MB, et al. Genetic stability of Brucella abortus isolates from an outbreak by multiple-locus variable-number tandem repeat analysis (MLVA16). BMC Microbiol. 2014;14:186.

19. Le Flèche P, Jacques I, Grayon M, Al Dahouk S, Bouchon P, Denoeud F, et al. Evaluation and selection of tandem repeat loci for a Brucella MLVA typing assay. BMC Microbiol. 2006;6:9.

Submit your next manuscript to BioMed Central and we will help you at every step:

- We accept pre-submission inquiries

- Our selector tool helps you to find the most relevant journal

- We provide round the clock customer support

- Convenient online submission

- Thorough peer review

- Inclusion in PubMed and all major indexing services

- Maximum visibility for your research

Submit your manuscript at www.biomedcentral.com/submit
Biomed Central 